\title{
Age Trends in Children's Time-sharing Performance
}

\author{
LEANN LIPPS BIRCH \\ University of Michigan
}

\begin{abstract}
To investigate developmental differences in time-sharing performance, 60 boys, 20 in each of three age groups (7-, 10-, and 13-year-olds) performed an auditory matching task and a tracking task alone and concurrently, the latter under two sets of instructions. Decrements produced by concurrent performance were compared for the three age groups. When the time-shared tasks were presented as equally important, time-sharing produced significantly greater proportional decrements in the tracking performance of the younger children and for all age groups tracking task decrements were directly related to matching task difficulty. Subsequently, the children were instructed that one or the other of the tasks was more important and that they were to improve their performance on that task. All three age groups showed a significant improvement on the task emphasized by instructions. The relationship of the results to two models of information processing is discussed.
\end{abstract}

That children improve their performance on a variety of activities with development is well known, but there are almost no data to indicate how concurrent performance of activities changes with development. The experiment reported here was designed to investigate developmental differences in how children timeshare when performing two concurrent tasks.

Two or more distinct activities are often performed at the same time, such as carrying on a conversation while walking or listening to music while reading. However, certain "biological bottlenecks" make it physically impossible to engage in certain activities concurrently; we cannot play the accordian while typing. Another limiting factor is less well understood but is related to the limits in the capacity for processing information.

Limited information processing capacity has been demonstrated by studies of time-sharing with adults in which the concurrent performance of two tasks produces decrements in one or both of the tasks relative to levels

This report is based on a dissertation submitted to the University of Michigan in partial fulfillment of the requirements for the Ph.D. degree. The research was supported in part by a Rackham dissertation grant. The author wishes to thank Patricia Miller for her advice and support throughout the project, and the children, teachers, and William Schongalla, Principal of Spencer Elementary School in Whimore Lake. Michigan, for their cooperation in the data collection. Special thanks go to David Birch for his very helpful comments and criticisms of this manuscript. A shorter version of this paper was presented at the biennial meeting of the Society for Research in Child Development, Denver, 1975. Requests for reprints should be sent to Leann Lipps Birch. Child Development Laboratory, School of Human Resources and Family Studies, University of Illinois, Urbana-Champaign, Illinois 61801. 
of performance attained on the tasks alone (Welch, 1898; McLeod, 1973). This finding has provided the basis for several limited capacity models of information processing.

In one model, Kahneman (1973) states that all cognitive processes demand processing capacity. If a task can be performed with less than the total capacity for mental effort, then the spare processing capacity can be allocated to the performance of a second task. Successful concurrent performance of a second task is interpreted within this model as indicating that adequate spare capacity has been allocated to the second task. Keele's (1973) model differs by stating that some but not all cognitive processes require space in a limited capacity mechanism. Decrements in time-sharing appear when two mental operations simultaneously require the mechanism. The interpretations of developmental differences in timesharing performance and their implications for cognitive development provided by these two models will be discussed in a later section.

Performance decrements produced in a second task in time-sharing by adults are often a positive function of the difficulty of the primary task (Johnston, Greenberg. Fisher, \& Martin, 1970; Posner \& Bois, 1971; Kantowitz \& Knight, 1974), where difficulty is defined by an error measure on the primary task in a baseline condition. The basic relationship has been replicated using a variety of primary tasks, including same-different letter matching (Posner \& Bois, 1971), memory tasks (McLeod, 1973), and a Fitts' tapping task at two levels of complexity (Kantowitz \& Knight, 1974). Different secondary tasks have also been used, including tracking (McLeod, 1973), simple reaction time (Posner \& Bois, 1971), and digit naming (Kantowitz \& Knight, 1974).

The value of the secondary task technique for evaluating primary task demands is illustrated by the time-sharing studies of Bahrick, Noble and Fitts (1954) and Bahrick and Shelly (1958). Their results showed that performance on a secondary task can reveal effects of practice on a primary task after asymptotic levels of performance on the primary task have been reached. This finding is consistent with the suggestion that, with practice, a task becomes easier as it requires less information processing for its performance (Mackworth, 1970). In the present study the secondary task technique was used to look for developmental trends, rather than for practice effects, in the relationship between primary task difficulty and secondary task decrements in time-sharing. It was assumed that the same tasks would be easier for older subjects.

In his review of developmental differences in the temporal limits of information processing, Wickens (1974) tentatively concluded that in addition to developmental differences in performance attributable to non-processing factors such as practice, motivation, and incentive, there are irreducible developmental differences in central information processing rates. Although data are scarce in some areas, developmental 
differences in central processing appear in a variety of experimental settings, including tachistoscope recognition, visual search, stimulus familiarity, sequential effects, and choice reaction time. These data suggest that there may be developmental differences in central processing limitations in a task setting where multiple stimuli are presented and multiple responses are required. The possibility that developmental differences in the extent to which children are able to respond to verbal instructions about task priorities in time-sharing also exists.

In a study with adults which systematically investigated the effects of verbal instructions about task priorities on the distribution of performance decrements in time-sharing, Murdock (1965) found that giving instructions to "concentrate primarily on" one or the other of the time-shared tasks produced distinct differences in the expected direction in the serial position curves of the time-shared memory tasks for the two instructional conditions. The data were interpreted as evidence for subjects trading off the two tasks, that is, improving on the "concentrate primarily on" task while allowing larger decrements on the nonemphasized task. In a later study, Murdock (1969) cautioned against his earlier interpretation and stated that the results may have been an artifact of the particular tasks used. These studies offer some equivocal evidence that adults are able to modify their time-shared performance in the instructed direction, but there are no data on whether children can use such instructions. The present study included conditions designed to provide such data.

\section{METHOD}

\section{Subjects}

The subjects were 60 boys, 20 each from the first-, fourth-, and seventh grades. The mean ages for the three groups were $6 \mathrm{yr} .11$ months, $10 \mathrm{yr} .1 \mathrm{month}$, and $13 \mathrm{yr}$. 1 month, respectively. All children were white, middle-class, and attended elementary school in Whitmore Lake, Michigan. All children had vision and hearing within the normal range. Two subjects from the youngest group were replaced, one for failure to perform one task and the other for failure to follow instructions. Each of the 20 subjects within an age group was randomly assigned to one of the two instructional conditions.

\section{Apparatus and Materials}

An auditory same-different matching task with two levels of difficulty served as the primary task. Level of difficulty was varied by using two different rules for the same-different judgments, based on the finding that, for adults, making judgments using a higher order rule is more difficult than making matches using physical identity (Posner \& 
Mitchell, 1967; Posner \& Bois, 1971). A one dimensional compensatory tracking task was used as the secondary task. To investigate developmental differences in the impact of verbal instructions on the distribution of decrements produced by timesharing, all children time-shared the auditory matching and tracking tasks under two sets of instructions.

Auditory matching tasks. The stimuli for the matching tasks were three-, four-, and five letter words from Thorndike and Lorge (1944) frequencies of 1-AA. Fifty-eight nouns were selected. Each noun could be classified as a member of one of four categories (12-16 words per category): animals, parts of the body, clothing, and food. The two difficulty levels of the auditory matching task were generated by changing the basis of the matches. In the phonemic identity condition, judgments were based on phonemic identity of the words, "Are the two words in the pair the same word?" In the category matching, judgments were based on category identity, "Are the two words the same kind of word?" For the phonemic condition, the stimulus pairs were constructed using words from all four categories. Half of the "different" pairs came from within a category and half from different categories. In the category condition the "different" pair words came from different categories and the "same" pair words came from within a category. The words in the "same" pairs were never phonemically identical. The order of the pairs was randomly determined in all lists, with the restriction that not more than three "same" or "different" pairs occurred in succession. Within a stimulus list no word appeared in more than three pairs. Each stimulus list contained 15 "same" and 15 "different" pairs.

In both types of auditory matching the stimulus pairs were presented at a rate of one pair every $2 \mathrm{sec}$, allowing 30 pairs to be given during each $1 \mathrm{~min}$ trial. Six different stimulus lists were constructed for the phonemic condition and nine lists for the category condition so that no child heard the same list more than once. All stimulus materials were recorded in the same female voice using a Sony Model $500 \mathrm{~T}$ tape recorder and presented binuarally through headphones.

A cassette recorder (Sony Model TC-80) was used to record all stimuli and responses during presentation of the matching tasks. The timing of the verbal responses was recorded on the event recorder channel of a Bausch and Lomb I ab Recorder (Model VOM-5) that was connected through a voice operated relay to the microphone attached to the headphones worn by the child.

Compensatory tracking task. A simplified Electronic Tracking Apparatus (SETA) of the type described by Gain and Fitts (1959a) was connected to a Tektronix Type 503 cathode ray oscilloscope with a $15 \mathrm{~cm}$ display screen.

The SETA was housed in two cabinets: an experimenter's console and a subject's console. The experimenter's console generated the problem 
input using a potentiometer linked to a synchronous motor driven rotary cam. This cam caused the cam follower to move over an arc of $1.27 \mathrm{~cm}$ and this rotated the potentiometer 180 degrees. The irregularly shaped cam generated a sinusoidal voltage output which can be described by the following equation:

where

$$
y=A(\sin \omega t+\sin 2 \omega t)
$$

$$
\omega=\frac{2 \pi}{T}, \quad T=10 \mathrm{sec} .
$$

Six complete cycles were presented in each 1 min trial. The subject's error was calculated for each trial by an analog computer element also located in the expermenter's console. The analog element consisted of three $\mathrm{K} 2 \mathrm{X}$ Philbrick operational amplifiers and their associated feedback components. The output of the component was the integration of the absolute value of the error voltage (integrated absolute error). (IAE) is thus calculated by continuously summing the absolute discrepancy between the problem input and the subject's output during each 1 min trial:

$$
\mathrm{IAE}=\frac{1}{T} \int_{0}^{T}\left|X_{d}-X\right| d t .
$$

The subject's console displaycd the discrepancy between the problem input and the subject's output on a zero center voltmeter with a range of $\pm 12.5 \mathrm{~V}$ located on the front panel. The subject's console was hidden from view by a plywood screen, however, and the error was displayed to the subject on the cathode ray tube (CRT) of the oscilloscope as a dot which moved horizontally. In performing the tracking task the child manipulated the control knob with his preferred hand in order to keep the moving dot on the target dot in the center of the screen. Rotating the control knob $180^{\circ}$ was sufficient to compensate for maximum variations in problem voltage. Control-display time delay was set at zero. Clockwise motion of the control resulted in movement of the dot on the CRT to the right. The subject's solution output was also recorded on the analog channel of a Bausch and Lomb Lab Recorder. The child was seated facing the CRT display that appeared in the $17.5 \times 19.0 \mathrm{~cm}$ rectangular opening of a large plywood pancl. The CRT display was approximately $45 \mathrm{~cm}$ from the subject and the height of the chair used was adjusted so that the display was near eye level for all subjects. The experimenter was seated to the left of the subject.

\section{Procedure}

The experiment was conducted during regular school hours in a trailer parked outside the school. Subjects were seen individually by the same female experimenter for one session about $1 \mathrm{hr}$ in length. Within the 
TABLE 1

Plan of Experimental Session

\begin{tabular}{|c|c|c|}
\hline \multirow{2}{*}{$\begin{array}{l}\text { Performance condition } \\
\text { and block number }\end{array}$} & \multicolumn{2}{|r|}{ Task } \\
\hline & Tracking & Auditory matching \\
\hline I Alone $(\mathrm{Tr})$ & \multicolumn{2}{|l|}{ Practice } \\
\hline 2 Alone $(\mathrm{Tr})$ & \multicolumn{2}{|l|}{ Practice } \\
\hline 3 Alone (Tr) & \multicolumn{2}{|l|}{$\mathrm{Tr}_{1}$} \\
\hline 4 Alone $\left(A_{w}\right)$ & & $A_{w}$-Phonemic matching \\
\hline 5 Time-shared & $\operatorname{Tr}$ & $A_{w}$ \\
\hline 6 Alone $(T r)$ & \multicolumn{2}{|l|}{$\mathrm{Tr}_{2}$} \\
\hline 7 Alone $\left(A_{c}\right)$ & & $\mathrm{A}_{\mathrm{c}}$-Category matching \\
\hline 8 Time-shared & $\operatorname{Tr}$ & \multirow[t]{2}{*}{$A_{c}$} \\
\hline 9 Alone (Tr) & $\operatorname{Tr}_{3}$ & \\
\hline \multicolumn{3}{|c|}{ Instructions } \\
\hline 10 Time-shared & $\operatorname{Tr}$ & \multirow[t]{2}{*}{$A_{c}$} \\
\hline 11 Alone $(\mathrm{Tr})$ & $\operatorname{Tr}_{4}$ & \\
\hline
\end{tabular}

session all children were given the same ordering of the tasks. A plan of the expermental session appears in Table 1.

Subjects initially received 10 tracking trials prior to the introduction of the auditory matching tasks. Trials 1 through 7 were classified as practice trials, Trials 8-10 constituted Block $\mathrm{Tr}_{1}$, Trials 14-16 Block $\mathrm{Tr}_{2}$, Trials 20-22 Block $\mathrm{Tr}_{3}$, and Trials 26-28 Block $\mathrm{Tr}_{4}$. Each tracking trial was $1 \mathrm{~min}$ in duration and the intertrial interval was approximately $15 \mathrm{sec}$. During this interval the subject was told his error score for the preceding trial. There were no regularly scheduled rest periods during this part of the session but if the child appeared tired or inattentive, or if his hand seemed to fatigue quickly, brief rests were given as necessary.

After the $\operatorname{Tr}_{1}$ block, the phonemic matching $\left(A_{p}\right)$ condition of the auditory task was introduced. First, three $1 \mathrm{~min}$ trials were presented alone, then three $A_{p}$ trials were given timeshared with tracking Trials 11-13 $\left(\operatorname{Tr}+A_{p}\right)$. Block $\operatorname{Tr}_{2}$ followed the first time-shared block. Tracking alone blocks were interspersed in this manner in order to obtain a running baseline. Performance on time-shared blocks was always compared with the immediately previous alone blocks so that any practice effects operated in the direction of reducing differences between the alone and time-shared conditions. After a 2 min rest interval, the category matching $\left(\Lambda_{c}\right)$ condition was presented alone for three trials, followed by time-sharing with tracking Trials $17-19\left(\operatorname{Tr}+A_{c}\right)$.

In both the time-shared blocks, $\operatorname{Tr}+A_{p}$ and $\operatorname{Tr}+A_{c}$, the tracking task and the auditory matching tasks were presented as equally 
important and the children were told to perform both tasks as well as possible. Two minute rest intervals were given after the $\mathrm{Tr}_{1}, \mathrm{Tr}_{2}$, and $\mathrm{Tr}_{3}$ blocks, during which the subject was encouraged to leave his seat and move about. All other intervals between trial blocks were approximately 30 sec long.

Following the $\operatorname{Tr}_{3}$ block, instructions designed to alter time-sharing performance were administered. At each age level the children were randomly assigned to one of the two instructional conditions in which either the tracking task was emphasized or the auditory matching task was emphasized. Special emphasis was given to one of the tasks in three ways. First, it was designated as more important; second, the children were told to pay more attention to it; and third, they were told to improve their performance on the emphasized task in comparison with their performance on the previous time-shared equal-emphasis condition. After the administration of the instructions, the children performed another block of category matching time-shared with the tracking task, $\operatorname{Tr}+\mathrm{A}_{\mathrm{c}}$, followed by the final tracking alone block, $\operatorname{Tr}_{4}$.

\section{RESULTS}

\section{Compensatory Tracking Task}

Because the baseline levels of performance on the tracking task alone were very different for the three age groups, performance decrements produced by time-sharing were compared relative to each group's baseline level. A logarithmic transformation was done on the IAE scores for each subject and the transformed scores were used in all analyses of the tracking data. The $1 \mathrm{n}$ transform eliminated the heterogeneity of variances that existed for the three age groups in the IAE data. By evaluating differences between the alone and time-shared conditions using the transformed $1 \mathrm{nIAE}$ scores, statements were made about proportional decrements, rather than absolute decrements produced by timesharing: $1 n\left(\operatorname{Tr}+A_{p}\right)-\ln (\operatorname{Tr})=\ln \left(\operatorname{Tr}+A_{p} / \operatorname{Tr}\right)$. This means, for example, that an increase in error score from 80 to 100 IAE units from the alone condition to the time-shared condition is equivalent to an increase from 20 to $25 \mathrm{IAE}$ units, because the ratios of the alone and time-shared scores are the same in the two cases. Perfect performance corresponded to an IAE score of zero and running the problem with no control response corresponded to a score of 5.65 InIAE units.

When the three age groups were compared on the basis of their tracking performance in the alone conditions, the older subjects showed consistently smaller error scores throughout the session. This is shown by the filled circles plotted in Fig. 1, where for example, the mean InIAE scores for the 7-, 10-, and 13-year-olds on Trial 10 can be seen to be approximately $4.2,3.7$, and 3.3 , respectively. A one-way analysis of 


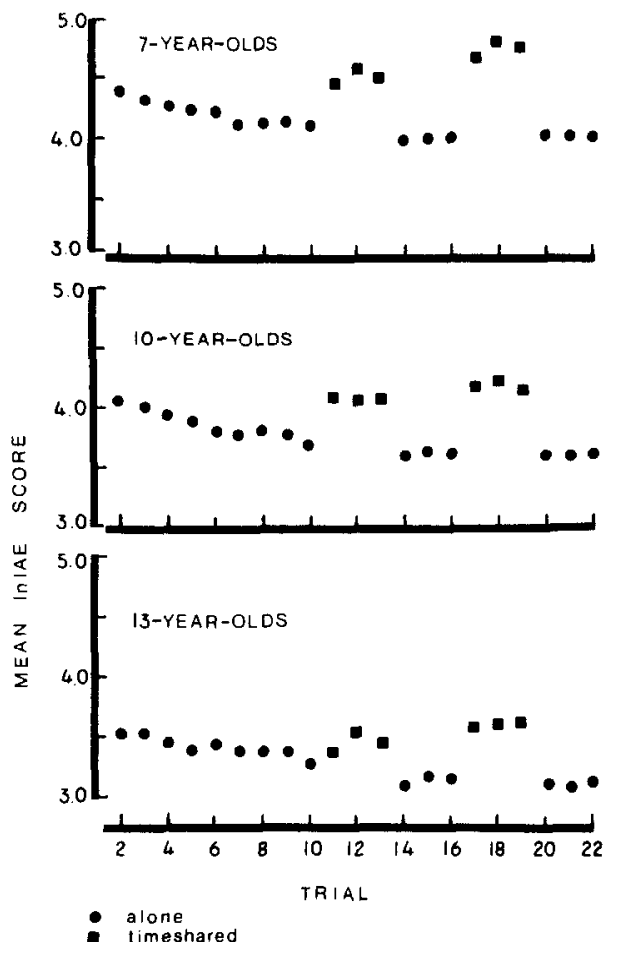

FIG. 1. Mean tracking score in InIAE units for Trials 2-22.

variance with age as the factor was done on the total $1 \mathrm{nIAE}$ score for each of the four tracking alone blocks $\left(\mathrm{Tr}_{1}, \mathrm{Tr}_{2}, \mathrm{Tr}_{3}, \mathrm{Tr}_{4}\right)$. The resulting $F$ values for all four analyses were significant at the $p<.001$ level. Pairwise comparisons on adjacent pairs of means were also significant at the $p<.001$ level. Baseline tracking performance is clearly different for the three age groups through the session.

The major focus of the experiment was on the question of developmental differences in time-sharing performance. To determine whether significantly greater proportional decrements existed in tracking performance as a consequence of time-sharing for the younger children, tracking decrement scores in logarithm units were obtained for each subject. The total InIAE score obtained on the immediately previous tracking alone block was subtracted from the total time-shared block 1nIAE score for both types of the auditory matching task: $D_{p}=\left(\operatorname{Tr}+A_{p}\right)-\left(\operatorname{Tr}_{1}\right) ; D_{c}=(T r$ $\left.+\mathrm{A}_{c}\right)-\left(\mathrm{Tr}_{2}\right)$.

For phonemic matching, the tracking decrement scores, $D_{p}$, for the 7-, 10 -, and 13-year-olds were $1.25, .90$. and .35 , respectively: For category matching, corresponding tracking decrement scores, $D_{c}$, were $2.31,1.73$, and 1.30. Both auditory matching tasks produced larger proportional decrements in the tracking performance of the younger groups and for all 
groups category matching produced greater proportional decrements in tracking performance than phonemic matching.

A $2 \times 3$ analysis of variance for repeated measures was performed on the obtained $D_{p}$ and $D_{c}$ tracking decrement scores with type of match as the repeated measure and age as the second factor. The main effect of age was significant, $[F(2,57)=7.98 ; p<.01]$, with larger proportional decrements in the performance of younger children. The main effect of type of auditory match was also significant $[F(1,57)=72.37 ; p<.001]$, with category matching producing greater decrements than phonemic matching. The age $\times$ type of match interaction was almost nonexistent $[F(2,57)=.33]$, indicating that the difference between the tracking decrements produced by category matching and phonemic matching was independent of the age of the children. Individual pairwise comparisons performed for each type of auditory matching task showed that for the $D_{p}$ scores, the 10-years, 13-years pair $[t(20)=2.07 ; p<.05]$ was significant, while the 7-years, 10 years pairs was not. Corresponding comparisons for the $\mathrm{D}_{\mathrm{c}}$ scores indicated that the 7-years, 10 -years pair $[t(20)=2.01 ; p<.05]$ was significant, while the 10-years, 13-years pair was not.

\section{Auditory Matching Tasks}

In analyzing the data from the auditory matching tasks, errors were defined to include both incorrect responses (i.e., saying "yes" when "no" was correct, or vice versa) and omissions. Predictably, the older children showed consistently smaller error scores on both types of auditory matching than the younger children. In the baseline alone conditions, the mean errors on the phonemic matching for the 7-, 10-, and 13-year-olds were $16.3,5.3$, and 3.9 , respectively. For the category matching task, the scores were 44.8, 30.4, and 15.9, respectively. These scores are plotted for each three-trial block in Fig. 2 using the solid lines. An error score of 90 was the maximum possible.

Figure 2 shows that time-sharing produced performance decrements in both types of auditory matching tasks for all age groups. The mean decrement scores for the 7-, 10-, and 13-year-olds on phonemic matching were $6.3,6.2$, and 3.1 , respectively, and for category matching they were $9.4,8.3$, and 4.4. Two two-way analyses of variance for repeated measures were done on the error scores with performance condition (alone or time-shared) and age as factors. The first analysis used the scores for the phonemic matching blocks, $A_{p}$ and $\operatorname{Tr}+A_{p}$, and the second the scores on the $A_{c}$ and $T r+A_{c}$ blocks. The main effect of performance condition was significant in both analyses: for phonemic matching, $F(1,57)=21.40, p$ $<.001$; and for category matching, $F(1,57)=34.40, p<.001$. Timesharing produced decrements in the performance of both phonemic category matching tasks. The effect of age was significant in both analyses, $A_{p}$, 


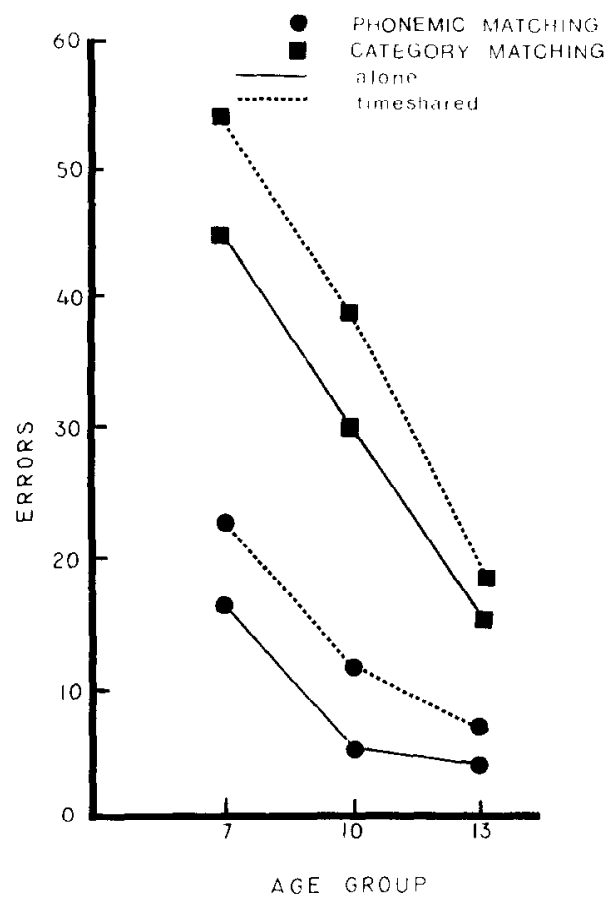

Fig. 2. Auditory matching errors as a function of age group.

$F(2,57)=24.01, p<.001 ; \mathrm{A}_{c}, F(2,57)=31.04, p<.001$, reflecting the consistently larger error scores of the younger children. The performance condition $\times$ age interaction did not reach significance in either analysis; both $F$ ratios were less than 1.0 , indicating that time-sharing the tracking task did not produce decrements in the performance of the matching tasks which differed as a function of age.

As shown in Fig. 2, the baseline levels of performance on auditory matching tasks alone differed for the three age groups (Box tests for homogeneity were not significant for the auditory match data). For this reason, the auditory matching data were also examined for developmental differences in proportional decrements produced by time-sharing. Neither of two one-way analyses of variance with age as the factor using the obtained proportional decrement scores for phonemic matching and category matching reached significance. Time-sharing does not produce proportional decrements in the performance of either auditory matching task which differ as a function of age.

Figure 2 also shows that more errors were made on category matching than phonemic matching for all age groups, and the difference between the error scores on the two types of matching tasks is greater for the younger children. Two $2 \times 3$ analyses of variance with type of matching task as the 
TABLE 2

Effects of Instructions on Timesharing. Mean InIAE Scores and Auditory Matching Errors for Tracking EMPHasis AND AUDITORY EMPHASIS GROUPS ${ }^{a}$

\begin{tabular}{|c|c|c|c|c|}
\hline \multirow[b]{3}{*}{ Age group } & \multicolumn{4}{|c|}{ Instructional condition } \\
\hline & \multicolumn{2}{|c|}{$\begin{array}{c}\text { Tracking emphasis } \\
\text { block }\end{array}$} & \multicolumn{2}{|c|}{$\begin{array}{l}\text { Auditory matching } \\
\text { emphasis block }\end{array}$} \\
\hline & $\begin{array}{c}\text { Equal } \\
\text { emphasis }\end{array}$ & $\begin{array}{l}\text { Tracking } \\
\text { emphasis }\end{array}$ & $\begin{array}{c}\text { Equal } \\
\text { emphasis }\end{array}$ & $\begin{array}{l}\text { Auditory } \\
\text { matching } \\
\text { emphasis }\end{array}$ \\
\hline \multicolumn{5}{|l|}{ 7-year-olds } \\
\hline InIAE score & 4.97 & 4.83 & $(4.82)$ & $(4.65)$ \\
\hline Auditory matching errors & $(52.0)$ & $(53.0)$ & 56.2 & 47.9 \\
\hline \multicolumn{5}{|l|}{ 10-year-olds } \\
\hline InIAE score & 4.30 & 3.99 & $(4.28)$ & $(4.44)$ \\
\hline Auditory matching errors & $(39.2)$ & $(36.5)$ & 37.8 & 38.0 \\
\hline \multicolumn{5}{|l|}{13 -year-olds } \\
\hline lnIAE score & 3.62 & 3.30 & $(3.73)$ & $(3.98)$ \\
\hline Auditory matching errors & $(22.7)$ & $(24.5)$ & 17.9 & 16.5 \\
\hline
\end{tabular}

${ }^{a}$ Nonemphasized task scores in parentheses.

repeated measure and age as the second factor were done on the error data. The first analysis used the scores obtained on the matching tasks alone. The effect of type of match was significant $[F(1,57)=291.6, p<.001]$, and so was the effect of age $[F(2,57)=45.10, p<.001]$. The interaction was significant at the $p<.001$ level, $[F(2,57)=15.40]$ going from phonemic matching to category matching produced less change in the error scores of the older children. A corresponding analysis of the time-shared scores showed very similar results. When the effects of type of match were also examined using proportional difference scores rather than absolute scores, the age differences no longer appear. Two one-way analyses of variance using the proportional difference scores obtained in both the alone and time-shared conditions were performed. Neither $F$ ratio approached significance. Going from phonemic matching to category matching produces a greater absolute difference in the error scores for younger children, but there is not difference with age in the proportional change in error scores produced by going from phenemic matching to category matching.

\section{Effects of Instructions on Time-sharing}

Half of the children at each age were randomly assigned to one of two instructional conditions. To determine that the two groups did not differ in 
their performance of either tracking or category matching prior to the adminstration of the differential instructions, two two-way analyses of variance with instructional condition and age as factors were done using the 1nIAE tracking scores and the auditory matching scores for the $\operatorname{Tr}+\mathrm{A}_{\mathrm{c}}$ block prior to the administration of the instructions. In both analyses, the $F$ ratios for instructional condition were less than 1.0.

Tracking-emphasis groups. When the tracking-emphasis $\operatorname{Tr}+\mathrm{A}_{\mathrm{c}}$ block scores were compared with the previous equal emphasis $\mathrm{Tr}+\mathrm{A}_{\mathrm{c}}$ block scores, the data show that all three age groups were able to improve their time-shared performance on the tracking task. The mean tracking and auditory matching scores for the tracking emphasis groups appear in Table 2.

A two-way repeated measures analysis of variance with instructional condition and age as factors was done using the $1 \mathrm{nIAE}$ tracking scores. The two levels of instructional factor were the equal emphasis condition of the previous $\operatorname{Tr}+\mathrm{A}_{\mathrm{c}}$ block and the $\mathrm{Tr}+\mathrm{A}_{\mathrm{c}}$ tracking-emphasized block. The main effects of instruction $[F(1,27)=31.0, p<.001]$ and age $[F(2,27)=23.6, p<.001]$ were significant, reflecting the better tracking performance of the older children in both blocks. The interaction was not significant $[F(2,27)=1.79]$, indicating that although the instructions were effective in producing performance changes in the desired direction, the impact of the instructions on performance was independent of age.

A corresponding analysis of the errors on the nonemphasized auditory matching task showed only a significant effect of age $[F(2,27)=16.09, p$ $<.001$ ] reflecting the generally better performance of the older subjects. Neither the effect of instruction nor the instruction $\times$ age interaction reached significance.

Auditory-emphasis groups. As shown in Table 2, the children in these groups were also able to use instructions to modify their time-sharing performance in the instructed direction.

The data of the auditory-emphasis groups were subjected to the same analyses as those of the tracking-emphasis subjects. The results of the two-way repeated measures analysis of variance of the auditory matching errors with instructional condition and age as factors showed both main effects were significant: instruction $F(1,27)=6.71, p<.05$; and age $F(2,27)=25.19, p<.001$. The interaction was significant for this group $[F(2,27)=4.54, p<.05]$ and in an unexpected form: The impact of the instructions was greatest for the youngest children.

The results of the corresponding analysis of the scores on the nonemphasized tracking task indicated that the effect of age was again significant $[F(2.27)=14.2, p<.001]$, reflecting the better performance of the older subjects. The interaction was also significant $[F(2,27)=9.08, p<$ $.01]$ although the main effect for instruction was not. The tracking error scores of the two older groups showed slight increases when auditory 
matching was emphasized whereas the youngest children actually reduced their error scores on the tracking task.

\section{DISCUSSION}

The results have shown that under time-sharing conditions the auditory matching tasks produced less impact on the tracking task performance of older children. When the decrements obtained in tracking performance for the three age groups were compared proportional to their respective baselines, the younger groups showed significantly larger time-sharing decrements. Because the baseline tracking error scores are greater for the younger subjects, this means that the younger groups also showed greater absolute decrements. Predictably, the older children made significantly fewer errors on the auditory matching tasks when they were preformed alone. This was true for both phonemic and category matching. The general relationship between primary task difficulty and secondary task decrements that has been noted in time-sharing studies using the secondary task technique with adults (Johnston et al., 1970; Kantowitz \& Knight, 1974) appears when the age groups are compared: decreasing error scores (signifying decreasing difficulty) with increasing age on the primary auditory matching tasks are accompanied by a corresponding decrease in proportional tracking decrements. This relationship was obtained for both phonemic and category matching tasks.

Developmental differences noted in the performance of the tracking and matching tasks in baseline alone conditions may be reflecting what Wickens (1974) has called nonprocessing factors: motivation, incentive, and practice. The obtained developmental differences in proportional tracking decrements produced by time-sharing suggest that, where nonprocessing factors can be considered to be controlled by using proprotional scores, greater central channel processing limitations may exist for younger children. This conclusion is consonant with the results of research on developmental differences in rapid information processing tasks reported by Wickens (1974). To begin to determine the nature and locus of these central processing differences, a more molecular analysis of time-sharing performance needs to be performed using temporal as well as error measures.

Category matching produced greater proportional decrements in tracking task performance than phonemic matching for all three age groups. The children in all three age groups also showed significantly larger error scores on category matching than phonemic matching, indicating that category matching was more difficult for all three groups. Once again, the direct relationship between primary task difficulty and secondary task decrements in time-sharing previously found in time-sharing studies with adults appears, this time in the data for each age group separately. For the 
children at each age level, making matches on a basis of a category rule (e.g., both are animals) was more difficult than matching on the basis of phonemic identity. Posner and Mitchell's (1967) adult subjects produced longer reaction times for rule matching and these longer reaction times were used as evidence for rule matching requiring relatively more information processing than identity matching.

The limited capacity models of information processing of Kaheman (1973) and Keele (1973) have not addressed the general question of how information processing demands of tasks might differ for children at various levels of cognitive development, but these models can provide alternative interpretations of the obtained developmental differences in time-sharing performance. The data of the present experiment do not provide support for one interpretation to the exclusion of the other.

1. The first interpretation is based on Kahneman's (1973) model. Man is conceptualized as having a limited general capacity for processing information. This general capacity is allocated to processes and activities in a flexible manner. All mental processes, whether perceptual-motor or coding-transformational, require the allocation of limited capacity. If an activity can be performed with the allocation of less than the total capacity for mental effort, the spare capacity can be allocated to another task. Successful concurrent performance of a second activity is interpreted within this model as an indication that the individual has allocted spare capacity to the performance of the second task. Therefore, the addition of a secondary task can serve as a diagnostic tool for evaluating the demands for attentional capacity made by a primary activity. The finding that the younger children show larger proportional decrements in the secondary tracking task than the older children is interpreted within this framework as indicating that for the younger child, the same auditory matching task demanded relatively more of the total capacity leaving relatively less spare capacity to devote to the performance of the secondary tracking task.

2. This alternative is suggested by Keele's (1973) formulation, in which some but not all mental operations require space in a limited capacity mechanism. Decrements in time-sharing appear when two mental operations simultaneously require the central mechansim and operations that do not require the mechanism can proceed in parallel with other operations without interference. In general, processes of response organization and execution are thought to make the greatest demands on central processing space, causing greater interference than perceptual processes. If children at different levels of cognitive development are using qualitatively different operations and strategies in performing the same time-shared tasks, then the performance differences in time-sharing are reflecting different patterns of interference produced by different process combinations.

Verbal instructions about task priorities proved to be a powerful factor in 
determining the locus of decrements in timesharing for all age groups. For the auditory-emphasis group, the improvement in auditory matching performance was greatest for the youngest children who also improved their nonemphasized tracking performance. Unlike Murdock's (1965) adult subjects, these 7-year-olds did not trade off larger decrements in the nonemphasized task for improved performance on the other task; only the 13-year-olds in this instructional condition appeared to be trading off one task for the other in the manner of Murdock's adults. Because every subject probably has some set of priorities about the relative importance of time-shared tasks even in the absence of specific verbal instructions, the present finidings underline the methodological importance of explicitly conveying the task priorities to the subject.

\section{REFERENCES}

Bahrick, H. P., Novel, M., \& Fitts, P. M. Extra task performance as a measure of learning a primary task. Journal of Experimental Psychology, 1954, 48, 298-302.

Bahrick, H. P., \& Shelly, C. Timesharing as an index of automatization. Journal of Experimental Psychology, 1958, 56, 288-293.

Gain, P., \& Fitts, P. M. A simplified electronic tracking apparatus. WADC Technical Report No. 44, 1959.

Johnston, W. S., Greenberg, S. N., Fisher, R. P. \& Martin, D. W. Divided attention: A vehicle for monitoring memory processes. Journal of Experimental Psychology, 1970, 83, $164-171$.

Kahneman, D. Attention and effort. Englewood Cliffs, New Jersey: Prentice-Hall, 1973.

Kantowitz, B. H., \& Knight, J. L. Testing tapping timesharing. Journal of Experimental Psychology, 1974, 103, 331-336.

Keele, S. W. Compatibility and time-sharing in serial reaction time. Journal of Experimental Psychology, 1967, 75, 529-539.

Keele, S. W. Attention and human performance. Pacific Palisades, California: Goodyear, 1973.

McLeod, P. D. Interference of "attend to and learn" tasks with tracking. Journal of Experimental Psychology, 1973, 99, 330-333.

Mackworth, J. F. Vigilance and attention. Middlesex: Penguin, 1970.

Murdock, B. B. Efrects of a subsidiary task on STM. British Journal of Psychology, 1965, 56, $413-419$.

Murdock, B. B. Measurement of retention of interpolated activity in STM. Journal of Verbal Learning and Verbal Behavior. 1969, 5, 469-472.

Posner, M. I., \& Boies, S. J. Components of attention. Psychological Review, 1971, 78, $391-408$.

Posner, M. I., \& Mitchell, R. F. Chronometric analysis of classification. Psychological Review, 1967, 74, 392-409.

Thorndike, E. L., \& Lorge, I. The teachers wordbook of 30,000 words. New York: Bureau of Publications, Teacher's College, Columbia University, 1944.

Welch, J. C. On the measurement of mental activity through muscular activity and the determination of a constant of attention. American Journal of Physiology, 1898, 1, 283-306.

Wickens, C. D. Temporal limits of human information processing: A developmental study. Psychological Bulletin, 1974, 81, 739-755. 